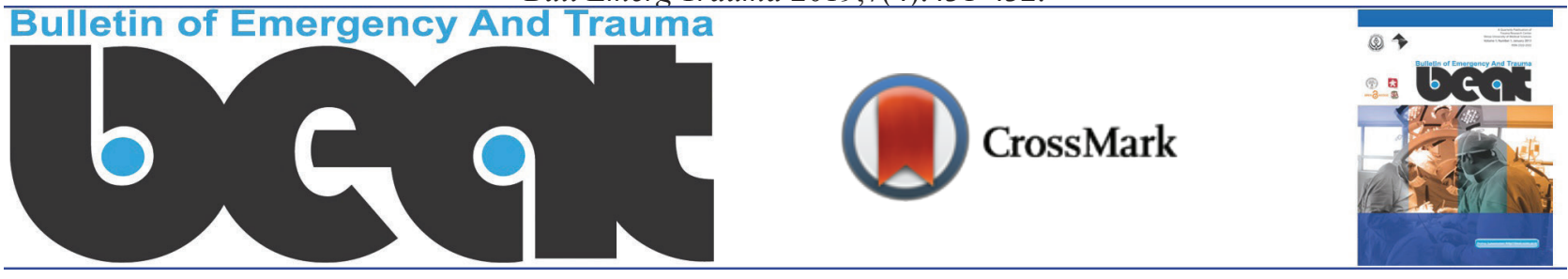

\title{
Search Strategy for Search Performance; Off-the-cuff or Being Sensitive
}

\author{
Behnaz Rastegarfar' ${ }^{1}$, Ali Ardalan ${ }^{1 *}$, Saharnaz Nejat ${ }^{2}$, Abbasali Keshtkar ${ }^{3}$, Mohammad Javad Moradian $^{4,5}$
}

\begin{abstract}
${ }^{1}$ Department of Disaster Public Health, School of Public Health, Tehran University of Medical Sciences, Tehran, Iran ${ }^{2}$ Department of Epidemiology and Biostatistics, School of Public Health, Tehran University of Medical Sciences, Tehran, Iran ${ }^{3}$ Department of Health Sciences Education Development, School of Public Health, Tehran University of Medical Sciences, Tehran, Iran ${ }^{4}$ Trauma Research Center, Shahid Rajaee (Emtiaz) Trauma Hospital, Shiraz University of Medical Sciences, Shiraz, Iran

${ }^{5}$ Department of Disaster and Emergency Health, School of Management and Medical Informatics, Shiraz University of Medical Sciences, Shiraz, Iran
\end{abstract}

*Corresponding author: Ali Ardalan

Address: School of Public Health, Tehran University of Medical Sciences, Poursina Ave. PO box: 14176-14411, Tehran, Iran. e-mail: aardalan@tums.ac.ir

Received: September 18, 2019

Accepted: September 29, 2019

\section{Dear Editor,}

We appreciate the interest of the authors in our article entitled "A productive proposed search syntax for health disaster preparedness research". They have rightly emphasized on the standard reporting of systematic reviews. However, as it is clear from the title and objective of the published article, we did not report results of a systematic review, our article instead aimed to present a syntax validation process which guide with creating a proper search strategy for systematic reviews on disaster preparedness [14]. As such neither a PRISMA flow nor an appraising tools were needed. Importance of having a proper search strategy for systematic reviews through a validation process has been widely mentioned in the literature [5-9]. This is crucial for optimizing the methodology of the systematic reviews and quality of the results accordingly. In regard with inclusion of non-English articles, we believed that the wealth of knowledge that exist in such articles should not have been missed. So we included them using the same eligibility criteria that were applied for English articles. Hand searching is a part of the validation process. We have selected the label "relative recall" to refer to the sensitivity index for the proposed syntax [10-12].

Our study presents a base search syntax with relative recall of 0.6 which can help researchers interested on disaster preparedness with finding as many as eligible studies possible [13]. It also helps the researchers to be more specific in case they need to focus on any specific hazard or alternative spelling/combination [1].

Conflicts of Interest: None declared. 


\section{References}

1. Rastegarfar B, Ardalan A, Nejat S, Keshtkar AA, Moradian MJ. A Productive Proposed Search Syntax for Health Disaster Preparedness Research. Bull Emerg Trauma. 2019;7(2):93-98.

2. Li L, Smith HE, Atun R, Tudor Car L. Search strategies to identify observational studies in MEDLINE and Embase. Cochrane Database Syst Rev. 2019;3:MR000041.

3. Thielen FW, Van Mastrigt G, Burgers LT, Bramer WM, Majoie H, Evers S, et al. How to prepare a systematic review of economic evaluations for clinical practice guidelines: database selection and search strategy development (part 2/3). Expert Rev Pharmacoecon Outcomes Res. 2016;16(6):705-721.

4. Eriksen MB, Frandsen TF. The impact of patient, intervention, comparison, outcome (PICO) as a search strategy tool on literature search quality: a systematic review. J Med Libr Assoc. 2018;106(4):420-431.

5. Montori VM, Wilczynski NL,
Morgan D, Haynes RB; Hedges Team. Optimal search strategies for retrieving systematic reviews from Medline: analytical survey. $B M J$. 2005;330(7482):68.

6. bir M, Bell SA, Puppala N, Awad O, Moore M. Setting Foundations for Developing Disaster Response Metrics. Disaster Med Public Health Prep. 2017;11(4):505-509.

7. Egan M, Maclean A, Sweeting H, Hunt K. Comparing the effectiveness of using generic and specific search terms in electronic databases to identify health outcomes for a systematic review: a prospective comparative study of literature search methods. BMJ Open. 2012;2(3).

8. Merrill JA, Orr M, Chen DY, Zhi Q, Gershon RR. Are We Ready for Mass Fatality Incidents? Preparedness of the US Mass Fatality Infrastructure. Disaster Med Public Health Prep. 2016;10(1):87-97.

9. Wilczynski NL, Haynes RB. Optimal search strategies for identifying diagnostic studies in EMBASE. BMC Med.

10. Wilczynski NL, Haynes RB; Hedges Team. EMBASE search strategies achieved high sensitivity and specificity for retrieving methodologically sound systematic reviews. $J$ Clin Epidemiol. 2007;60(1):29-33.

11. Durão $S$, Kredo $T$, Volmink J. Validation of a search strategy to identify nutrition trials in PubMed using the relative recall method. $\mathrm{J} \mathrm{Clin}$ Epidemiol. 2015;68(6):610-6.

12. Sampson M, Zhang L, Morrison A, Barrowman NJ, Clifford TJ, Platt $\mathrm{RW}$, et al. An alternative to the hand searching gold standard: validating methodological search filters using relative recall. $B M C$ Med Res Methodol. 2006;6:33.

13. Wilczynski NL, Haynes RB. Optimal search strategies for detecting clinically sound prognostic studies in EMBASE: an analytic survey. $J \mathrm{Am}$ Med Inform Assoc. 2005;12(4):481-5.

\section{Open Access License}

All articles published by Bulletin of Emergency And Trauma are fully open access: immediately freely available to read, download and share. Bulletin of Emergency And Trauma articles are published under a Creative Commons license (CC-BY-NC). 